\title{
Mumps Associated Acute Motor Axonal Polyneuropathy
}

\author{
Siyaram Didel $^{1} \cdot$ Renu Suthar ${ }^{1} \cdot$ Debasish Sahoo $^{1} \cdot$ Mini P. Singh $^{2} \cdot$ Jitendra K. Sahu $^{1}$
}

Received: 28 November 2016 / Accepted: 6 April 2017 /Published online: 24 April 2017

(C) Dr. K C Chaudhuri Foundation 2017

To the Editor: Aseptic meningitis is the most common central nervous system (CNS) complication of mumps infection. Asymptomatic pleocytosis is seen in $40-60 \%$ patients with mumps parotitis; meningitis in $15 \%$ and encephalitis in $0.1 \%$ $[1,2]$. CNS involvement may precede, co-exist or follow parotid swelling. We report a case of mumps encephalitis developing an unusual complication. A partially immunized 8-y-old boy presented with a history of bilateral parotid swelling with odynophagia following a brief febrile illness. He developed intractable vomiting, seizures, status epilepticus on day 5 of illness and subsequently remained in encephalopathic state. Paucity of movement was noted from second week of illness. Lumbar cerebrospinal fluid (CSF) showed lymphocytic pleocytosis, normal glucose and protein. MRI brain was normal. On examination he was wasted, encephalopathic, Glasgow Coma Scale was E4(meaningless)M4V1, had flaccid quadriparesis and generalized areflexia. His younger sibling also had a self-limiting febrile illness with bilateral parotitis. Nerve conduction study showed severe motor predominant axonal polyneuropathy with sensory sparing. IgM ELISA for mumps was positive in both the siblings. The child was managed conservatively with supportive care and he made a complete recovery.

In addition to mumps encephalitis, course of the index child was complicated with flaccid quadriparesis secondary to acute motor axonal polyneuropathy. It is a rare complication

Renu Suthar

drrenusuthar@gmail.com

1 Department of Pediatrics, Postgraduate Institute of Medical Education and Research, Chandigarh 160012, India

2 Department of Virology, Postgraduate Institute of Medical Education and Research, Chandigarh, India of mumps, only a few cases of Gullian barre syndrome; overlap with transverse myelitis have been reported [2,3]. Isolated cases of cranial nerve palsies including optic, facial, trigeminal and oculomotor nerves have been described. Type of neuropathy in mumps is sensory motor axonopathy, and the proposed pathogenesis may be related to molecular mimicry or with direct viral involvement [4].

A critical illness associated polyneuropathy (CIP) was another consideration, however he was never ventilated and risk factors for CIP were absent. Early onset axonal polyneuropathy and complete recovery within 1 mo of illness favors the diagnosis of mumps associated polyneuropathy in the index child. Mumps is an important cause of encephalitis in children and presence of bilateral parotitis points to the diagnosis. Acute motor axonal polyneuropathy is a rare and reversible complication of mumps virus infection.

\section{Compliance with Ethical Standards}

Conflict of Interest None.

Source of Funding None.

\section{References}

1. Koskiniemi M, Donner M, Pettay O. Clinical appearance and outcome in mumps encephalitis in children. Acta Paediatr Scand. 1983;72:603-9.

2. Bajaj NP, Rose P, Clifford-Jones R, Hughes PJ. Acute transverse myelitis and Guillain-Barre overlap syndrome with serological evidence for mumps viraemia. Acta Neurol Scand. 2001;104:239-42.

3. Bouskraoui M, Zineddine A, Najib J, Abid A. Association of Guillain-Barre syndrome and mumps in a 13-month-old infant. Arch Pediatr. 1998;5:928.

4. Triquenot A, Renard J, Lefevre M, et al. Acute axonal polyradiculoneuritis in mumps. Presse Med. 1996;25:1652. 